\title{
Kernos
}

Revue internationale et pluridisciplinaire de religion grecque antique

18 | 2005

Varia

\section{Barbara GofF, Citizen Bacchae. Women's Ritual Practice in Ancient Greece}

\section{Anne-Françoise Jaccottet}

Édition électronique
URL : http://journals.openedition.org/kernos/1535

DOI : 10.4000/kernos. 1535

ISSN : 2034-7871

\section{Éditeur}

Centre international d'étude de la religion grecque antique

\section{Édition imprimée}

Date de publication : 1 janvier 2005

Pagination : 531-533

ISSN : 0776-3824

\section{Référence électronique}

Anne-Françoise Jaccottet, «Barbara goff, Citizen Bacchae. Women's Ritual Practice in Ancient Greece », Kernos [En ligne], 18 | 2005, mis en ligne le 24 mai 2011, consulté le 21 septembre 2020. URL : http://journals.openedition.org/kernos/1535; DOI : https://doi.org/10.4000/kernos.1535 
Kernos 18 (2005), p. 531-574.

\section{Revue des Livres}

\section{Comptes rendus et notices bibliographiques}

Barbara Goff, Citizen Bacchae. Women's Ritual Practice in Ancient Greece, Berkeley / Los Angeles / London, University of California Press, 2004. 1 vol. $16 \times 23,5 \mathrm{~cm}, \mathrm{XII}+400$ p., 11 fig. ISBN : 0-520-23998-9.

L'ouvrage dense de Barbara Goff nous invite à nous plonger dans l'univers rituel des femmes de la Grèce ancienne, et plus particulièrement de l'Athènes des $\mathrm{v}^{\mathrm{e}}$ et $\mathrm{IV}^{\mathrm{e}}$ siècles av. J.-C. Plus fidèle à son sous-titre qu'à son titre principal, ce livre a en effet pour objet la reconstruction détaillée de la sphère d'action rituelle féminine et vise à faire (ré)apparaître le rôle à la fois de sujet et d'objet que les femmes ont endossé dans les divers rituels auxquelles elles ont été associées. S'articulant en cinq grands chapitres, l'ouvrage propose autant d'approches différentes de l'activité rituelle féminine.

En répondant aux trois questions matérielles qu'évoque le sujet (où? quand? quoi ?), le premier chapitre («Working toward a material presence ») fournit une base de travail bienvenue qui permet de mieux évaluer la place prise par les rituels féminins dans la vie quotidienne des Grecs de l'époque classique. Au catalogue des rites publics qui scandent l'année rituelle, B. Goff a eu l'heureuse initiative de joindre les rites accomplis dans la sphère privée de l'oikos (offrandes des femmes enceintes, rites entourant la naissance, le mariage et les funérailles). Le tableau de l'activité rituelle féminine se révèle ainsi multiple et fort dense, ce qui conduit l'A. à conclure que la participation des femmes aux divers rituels leur permet de construire une présence forte et complexe dans la vie de la communauté tout en leur apportant une certaine indépendance d'action, notamment financière (rôle de prêtresses et dédicaces d'offrandes).

Dans un second volet (« Ritual management of desire. The reproduction of sexuality »), l'A. s'interroge sur la façon dont les pratiques rituelles ainsi définies agissent sur la subjectivité des femmes. Fondé essentiellement sur l'étude de leur relation à la sexualité et au désir (leur propre désir comme celui qu'elles suscitent au travers du regard des hommes), ce chapitre tente de montrer comment les diverses fêtes religieuses féminines qui jalonnent l'année rituelle offrent aux femmes une palette d'expressions différentes de ce rapport à la sexualité, allant de la chasteté à la licence, avec pour résultat, selon l'A., le conditionnement de la femme à la place que la société patriarcale lui assigne: en dramatisant les identifications contradictoires qu'on attend d'elles, les rituels féminins soulignent la relation réciproque de sujet et d'objet que les femmes endossent simultanément dans les rituels, et qui les mène à développer une subjectivité notamment sexuelle plus utile aux autres qu'à elles-mêmes.

Le troisième chapitre ( $I n$ and out the city. Imaginary citizens ») explore quant à lui les rôles officiels et quasi politiques que certains rituels permettent aux femmes d'atteindre à certaines conditions. En analysant les mythes qui mettent en scène le sacrifice, salvateur pour la cité, d'une femme - plus particulièrement d'une parthenos - 
aussi bien que les occasions dans lesquelles les femmes agissent rituellement " pour la sauvegarde de la cité », l'A. insiste sur la possibilité, pour les femmes, d'accéder au travers du rituel à un rôle quasi politique; mais c'est paradoxalement la reconnaissance de l'altérité de la femme qui lui ouvre les portes de l'officialité; c'est parce qu'elle est toujours considérée comme extérieure à la société masculine que la femme peut lui apporter par le biais rituel des éléments constitutifs essentiels. Les divers rituels offrent ainsi à la femme une certaine expérience de la citoyenneté, relevant plus de l'imaginaire que d'une quelconque fonction réelle et qui occulte partiellement l'exclusion que les femmes ont à subir dans la société et la vie quotidienne.

Les deux derniers volets de cet ouvrages proposent des analyses plus spéculatives sur la question de la représentation de la femme, par elle-même et par la société masculine. Le chapitre 4 («Representing women. Ritual as a cultural resource») évoque la possibilité de l'existence d'une subculture féminine, focalisée sur le rituel, qui serait décelable dans trois domaines, la poésie féminine, la représentation des femmes et des rituels féminins sur la céramique attique et le ménadisme. Si, de façon attendue, l'A. pose plus de questions qu'elle ne propose de conclusion, elle voit tout de même dans ces trois créneaux l'émergence timide d'une voix plus proprement féminine qui emprunte les chemins du rituel pour mieux se donner une identité privée: l'existence de communautés rituelles facilite l'apparition de femmes poètes (Sappho, Nossis), alors que ces poètes au féminin se concentrent sur les ressorts du rituel pour obtenir une voix publique et représenter leur propre autorité poétique; la céramique quant à elle ne représente peut-être pas une voix indépendante et résistante de la part de la femme « historique », mais en tout cas une voix « privée » ou domestique qui ne correspond pas tout à fait avec l'idéologie officielle; le ménadisme enfin offre aux femmes une certaine expression d'autorité qui les met en état de négocier leur subordination et peut-être d'y résister psychologiquement. Le rituel se définit ainsi, non tellement comme le lieu de l'expression personnelle et autonome des femmes, ni comme celui de leur simple exploitation par la société patriarcale, mais comme le lieu d'une dialectique jamais donnée d'avance qui doit se construire constamment pour obtenir le consentement des femmes à un système qui n'est pas à leur avantage.

Le dernier chapitre («Women represented. Ritual in drama ») propose un parcours chronologique des tragédies et comédies conservées dans lesquelles les femmes sont représentées dans un rôle rituel. Sans proposer de véritable conclusion, l'A. souligne l'évolution qu'elle décèle entre les premières pièces d'Eschyle et les dernières d'Euripide ou encore d'Aristophane : le rôle rituel des femmes passe d'une phase de conflit ouvert et dramatisé avec la cité à une sorte de coopération raisonnée pour retrouver à l'extrême fin de la production «classique » un mode conflictuel d'opposition.

On l'aura sans doute compris, le livre de B. Goff est un ouvrage à thèse, un ouvrage engagé théoriquement et politiquement. S'appuyant sur le comparatisme anthropologique et avant tout sur la littérature féministe, l'A. s'applique à relativiser les options contradictoires souvent évoquées à propos de la sphère rituelle féminine, selon lesquelles la religion est soit le lieu de l'assujettissement idéologique et matériel des femmes à la société patriarcale qui les contrôle, soit inversement le lieu de l'expression de leur résistance à cet ordre masculin de la société. L'on saura gré à B. Goff d'avoir su proposer une interprétation plus nuancée, faisant de la sphère rituelle féminine le lieu d'une dialectique toujours remise en question entre l'oppression dont les femmes sont les victimes et la créativité que les espaces rituels leur offrent. S'appliquant à teinter les propos féministes d'une vision matérialiste, elle 
s'attache à montrer comment les femmes, bien que sujets des activités rituelles qu'elles mettent en œuvre, sont à la fois et réciproquement objets de l'idéologie masculine qui façonne leur identité féminine ("Women act and are acted upon in the same gesture », p. 59).

Mais le souci théorique qui se manifeste tout au long de l'ouvrage ne va pas sans laisser des traces, dommageables, dans la conception de cette recherche. Occupée à former un modèle théorique et à en prouver la pertinence dans le domaine rituel féminin, l'A. me paraît avoir engagé sa recherche à rebours, comme si les conclusions précédaient l'analyse. Cette impression qui prend corps au fil des pages semble trouver un reflet dans la structure des divers chapitres : commençant chaque volet de cette étude par une introduction théorique, souvent circonstanciée, l'A. propose en premier lieu, pour les 5 chapitres, un parcours des différentes théories et un résumé de la dialectique qu'elle-même voit dans la question; l'analyse des rituels ne vient que dans un second temps, en quelque sorte en guise d'argumentation et de preuve du bien-fondé des propos théoriques initiaux. Cette prédominance théorique se révèle aussi par l'importance prise par la littérature secondaire au détriment des documents antiques, comme si cette étude devait avant tout prendre une place dans le discours moderne sur les femmes plutôt que dans la réalité antique de la condition féminine. La voix que B. Goff veut rendre aux femmes antiques ressemble trop souvent à la voix des féministes contemporaines. Cette théorisation à outrance et ce regard de deuxième main sur bien des documents amène certaines distorsions que plus d'un exégète de l'antiquité aura du mal à concevoir : les Adonies, avec leur jardins portatifs que les femmes font germer puis laissent délibérément brûler sur les terrasses, sont par exemple interprétées comme la métaphore de la position de la femme dans la société : les jardins brûlés avant leur épanouissement et leur maturation évoquent, selon l'A. l'incapacité des femmes à être positivement productives et assied ainsi, tout en le constituant, le regard négatif que les hommes ont de la femme. La figure de la femme au miroir sur les vases attiques, pour prendre un autre exemple, peut bien être interprétée comme l'image d'elle-même que la femme préfère et qu'elle concourt à imposer sur les vases qui la concernent directement, ou encore comme la mise en abyme du regard de la femme sur elle-même; mais lorsque ce discours s'appuie sur l'image d'un médaillon de coupe, vase on ne peut plus masculin, présenté en illustration, l'impression de l'utilisation des documents pour servir une cause théorique plutôt comme centre véritable de l'analyse s'impose désagréablement à l'esprit du lecteur.

Retenons tout de même que l'ouvrage de Barbara Goff a le mérite - et peut-être la témérité - de prendre en compte un très vaste domaine de recherche, qu'il est intéressant de voir traité de façon unitaire. Si les positions théoriques souvent forcées de l'A. ne peuvent emporter l'adhésion de tout un chacun, au moins ce livre ne laissera-t-il pas le lecteur indifférent et le forcera-t-il à prendre position, sur les questions méthodologiques comme sur la problématique, très à la mode aujourd'hui, de l'identité féminine dans l'Antiquité.

Anne-Françoise Jaccottet

(Université de Lausanne)

Jérôme LAURent (éd.), Les dieux de Platon. Actes du colloque organisé à l'Université de Caen Basse-Normandie les 24, 25 et 26 janvier 2002, Université de Caen Basse-Normandie, Presses Universitaires de Caen, 2003. 1 vol. $14 \times 21,5 \mathrm{~cm}, 298$ p. ISBN : 2-84133-199-7. 